\title{
CARACTERIZAÇÃO NUTRICIONAL E COMPOSTOS ANTIOXIDANTES EM RESIIDUOS DE POLPAS DE FRUTAS TROPICAIS
}

\author{
Nutritional characterization and antioxidant compounds in pulp residues of tropical fruits
}

\author{
Mariana Séfora Bezerra Sousa ${ }^{1}$, Luanne Morais Vieira ${ }^{2}$, Manoel de Jesus Marques da Silva ${ }^{1}$, Alessandro de Lima ${ }^{3}$
}

\section{RESUMO}

O Brasil é um dos países que mais produz resíduos agroindustriais, como os resíduos de frutas pelas indústrias de polpas, o que tem contribuído para o aumento da produção do lixo orgânico, provocando graves problemas ambientais. Nesse contexto, estudos têm sido conduzidos com o intuito de investigar o valor nutricional desses resíduos, valorizando-os e sugerindo novas alternativas de utilização. Assim, neste trabalho, objetivou-se realizar a caracterização nutricional e determinar os compostos antioxidantes dos resíduos de polpas de frutas tropicais: acerola (Malpighia glabra L.), goiaba (Psidium Guayaba L.), abacaxi (Ananas comosus L.), cupuaçu (Theobroma grandiflorum), bacuri (Platonia insignis) e graviola (Annona muricata L.). Os resultados encontrados mostraram que os resíduos analisados apresentaram quantidades significativas de macronutrientes (carboidratos, proteínas e lipídios). Todos os resíduos avaliados, com exceção do cupuaçu, apresentaram valores elevados de vitamina C. Quanto aos carotenóides, destacou-se o resíduo de acerola com $881,56 \pm 9,01 \mu \mathrm{g} / 100 \mathrm{~g}$ e o resíduo de goiaba, com $644,9 \pm 10,02 \mu \mathrm{g} / 100 \mathrm{~g}$. Os resíduos analisados apresentaram baixas concentrações de antocianinas e flavonóides. Com relação aos teores de fenólicos totais se destacou o resíduo da polpa de acerola com 247,62 $\pm 2,08 \mathrm{mg} / 100 \mathrm{~g}$. Portanto, pode-se concluir que os resíduos de polpas de frutas empregados neste estudo são fontes potenciais de macronutrientes e compostos bioativos, destacando-se os resíduos de acerola e goiaba como mais ricos em compostos antioxidantes.

Termos para indexação: Nutrientes, compostos fenólicos, carotenóides, vitamina C.

\begin{abstract}
Brazil is one of the largest agro-industrial residues producers, such as waste fruit pulp industries, which has contributed to the increased production of organic waste, causing serious environmental problems. In this context, studies have been conducted in order to investigate the nutritional value of these wastes, valuing them and suggesting new alternatives for use. The objective of this study was to characterize and determine nutritional antioxidant compounds of the waste pulp of the following tropical fruits: acerola (Malpighia glabra L.), guava (Psidium Guayaba L.), pineapple (Ananas comosus L.), cupuaçu (Theobroma grandiflorum), bacuri (Platonia insignis) and soursop (Annona muricata L.). The results showed that the waste analyzed showed significant amounts of macronutrients (carbohydrates, proteins and lipids). All waste assessed, except cupuaçu, showed elevated levels of vitamin C. As for carotenoids, highlighted the residue of acerola with $881.56 \pm 9.01 \mu \mathrm{g} / 100 \mathrm{~g}$ and residue of guava, with $644.9 \pm 10.02 \mu \mathrm{g} / 100 \mathrm{~g}$. The waste also showed low concentrations of anthocyanins and flavonoids. Regarding of total phenolic levels, the residue of the pulp acerola with $247.62 \pm 2.08 \mathrm{mg} / 100 \mathrm{~g}$ of total phenolics was high. Therefore, it may be concluded that the waste of fruit pulp used in this study are potential sources of nutrients and bioactive compounds, especially the waste from acerola and guava as richer in antioxidant compounds.
\end{abstract}

Index terms: Nutrients, phenolic compounds, carotenoids, vitamin C.

(Recebido em 27 de abril de 2010 e aprovado em 11 de novembro de 2010)

\section{INTRODUÇÃO}

A indústria de alimentos, em especial a de processamento de frutos, produz ao longo de sua cadeia produtiva uma grande quantidade de resíduos agroindustriais, o que gera perda de divisas, além de inúmeros problemas ambientais (Sena \& Nunes, 2006). Atualmente, as agroindústrias investem no aumento da capacidade de processamento, gerando grandes quantidades de subprodutos, que em muitos casos são considerados custo operacional para as empresas ou fonte de contaminação ambiental (Lousada Junior et al., 2005).

Segundo Martins \& Faria (2002), calcula-se que, do total de frutas processadas, sejam gerados, na produção de sucos e polpas, entre 30 a $40 \%$ de resíduos agroindustriais. Como a quantidade de resíduos pode chegar a muitas toneladas, agregar valor a esses produtos é de interesse econômico e ambiental, necessitando de investigação científica e tecnológica,

\footnotetext{
${ }^{1}$ Instituto Federal de Educação, Ciência e Tecnologia do Piauí/IFPI - Teresina, PI

${ }^{2}$ Universidade Federal do Piauí/UFPI - Teresina, PI

${ }^{3}$ Instituto Federal de Educação, Ciência e Tecnologia do Piauí/IFPI - Avenida Pedro Freitas - 1020 - São Pedro - $64001-010$ - Teresina, PI alessandro@cefetpi.br
} 
que possibilite sua utilização eficiente, econômica e segura. (Schieber et al., 2001).

Os principais resíduos gerados no processamento de polpas de frutas são, dependendo do tipo da fruta processada, casca, caroço ou sementes e bagaço. Esses resíduos possuem em sua composição vitaminas, minerais, fibras e compostos antioxidantes importantes para as funções fisiológicas. No entanto, na maioria das fábricas, são desperdiçados (Matias et al., 2005). Tais resíduos poderiam ser utilizados, minimizando o desperdício de alimentos e gerando uma nova fonte alimentar.

Estudos recentes têm demonstrado que as frutas são ricas em muitos nutrientes e compostos antioxidantes, e que esses constituintes se concentram majoritariamente nas cascas e sementes (Costa et al., 2000; Melo et al., 2008; Abrahão et al., 2010). Vários autores têm associado os efeitos benéficos, à saúde do homem, do consumo regular de frutas, vegetais e grãos à presença de substâncias antioxidantes, como os compostos fenólicos, a vitamina $\mathrm{C}$ e os carotenóides (Vasconcelos et al., 2006; Kim et al., 2007; Pieniz et al., 2009).

Portanto, considerando as elevadas taxas de produção de resíduos agroindustriais gerados a partir das frutas e a importância dos antioxidantes para saúde da população, este estudo visou a caracterizar nutricionalmente os resíduos de polpas de frutas tropicais bem como quantificar os principais compostos antioxidantes, com a perspectiva de uma melhor utilização dos mesmos, do ponto de vista tecnológico e nutricional, agregando valor aos resíduos e aos seus produtos.

\section{MATERIAL E MÉTODOS}

\section{Material}

As amostras de resíduos de polpas de fruta foram doadas por uma indústria de polpa de fruta, localizada na cidade de Teresina - Piauí. Foram coletados, de uma única vez, $8 \mathrm{~kg}$ de resíduo de cada variedade de polpa de fruta, totalizando $48 \mathrm{~kg}$ de resíduos agroindustriais, que foram imediatamente transportados em caixas isotérmicas para o Laboratório de Alimentos do Instituto Federal de Educação, Ciência e Tecnologia do Piauí - IFPI, onde foram armazenados sob congelamento a $-18^{\circ} \mathrm{C}$ até o momento das análises. Esses materiais sofreram quarteamentos sucessivos até a obtenção de cerca de $400 \mathrm{~g}$ de cada resíduo que foram triturados em moinho analítico e armazenados sob congelamento a $-18^{\circ} \mathrm{C}$ até a realização dos ensaios analíticos.

Os resíduos de polpas de fruta congeladas utilizados neste trabalho foram de Acerola (Malpighia glabra L.),
Goiaba (Psidium Guayaba L.), Abacaxi (Ananas comosus L.), Cupuaçu (Theobroma grandiflorum), Bacuri (Platonia insignis) e Graviola (Annona muricata L.).

\section{Métodos}

\section{Determinação da composição nutricional}

A determinação de umidade foi feita pela secagem em estufa a $105^{\circ} \mathrm{C}$ até peso constante. A determinação do resíduo mineral fixo (cinzas) foi realizada pela incineração em mulfla a $550^{\circ} \mathrm{C}$. As proteínas foram determinadas pelo método de Kjeldahl, utilizando o fator de 6,25 para conversão do nitrogênio em proteína. Os lipídeos totais foram obtidos com extração da fração etérea por fluxo intermitente, utilizando éter etílico como solvente sob refluxo, em aparelho de Soxhlet. Os carboidratos foram obtidos por diferença. Todas as análises foram realizadas em triplicata e segundo as normas do Instituto Adolf Lutz (2005). Calculou-se o valor energético total (VET), considerando-se os fatores de conversão de $4 \mathrm{kcal} / \mathrm{g}$ de proteína, $4 \mathrm{kcal} / \mathrm{g}$ de carboidrato e $9 \mathrm{kcal} / \mathrm{g}$ de lipídeo, conforme Ferreira \& Graça (1983).

\section{Determinação de compostos antioxidantes}

Os compostos antioxidantes determinados nos resíduos de polpas de frutas estudados foram vitamina $\mathrm{C}$, carotenóides, flavonóides, antocianinas e fenólicos totais. $\mathrm{O}$ teor de vitamina $\mathrm{C}$ foi determinado por meio do método iodométrico (Instituto Adolf Lutz, 2005), em que se titulou a amostra com iodato de potássio a $10 \%$, obtendo-se o teor de vitamina $\mathrm{C}$ em miligramas a partir do volume gasto na titulação.

A determinação dos carotenóides foi realizada segundo Association of Official Analytical Chemists AOAC (2000), a extração foi realizada com álcool isopropílico e hexano e a leitura do extrato, em triplicata, em espectrofotômetro (Coleman 33 D) a $450 \mathrm{~nm}$. O resultado foi expresso em mg de carotenóides/100 g amostra

As antocianinas foram avaliadas segundo o protocolo da AOAC (2000). Foi utilizado como solução extratora etanol $90 \%$ e ácido clorídrico $1,5 \mathrm{~N}$, na proporção de 85:15. Após 24 horas de repouso (período de extração), o extrato foi filtrado. Alíquotas foram recolhidas para leitura a $535 \mathrm{~nm}$ em espectrofotômetro (Coleman 33 D), obtendose o teor de antocianinas em $\mathrm{mg} / 100 \mathrm{~g}$.

A análise dos flavonóides foi realizada por Cromatografia Delgada segundo Instituto Adolf Lutz (2005). Utilizou-se placa de sílica gel 60c, Merk, sem detector de fluorescência e com $0,2 \mathrm{~mm}$ de espessura e acetato de etila, metanol e ácido acético (25:1:1) como 
solventes. O padrão utilizado foi a quercetina $(0,1 \mu \mathrm{g}$ de flavonóides/g produto). O resultado foi expresso em $\mu \mathrm{g}$ de flavonóides/g amostra.

A determinação dos fenólicos totais seguiu a metodologia descrita por Swain \& Hills (1959). Para a preparação dos extratos foram utilizados $100 \mathrm{~mL}$ de água destilada e $20 \mathrm{~g}$ de resíduo de polpa de fruta. Do extrato aquoso de cada amostra, tomou-se $0,5 \mathrm{~mL}$ e adicionou-se $8 \mathrm{~mL}$ de água destilada e $0,5 \mathrm{~mL}$ do reagente Folin Ciocalteau (Merck). A solução foi homogeneizada e, após $3 \mathrm{~min}$, acrescentou-se $1 \mathrm{~mL}$ de solução saturada de $\mathrm{NaCO}_{3}$. Decorrida 1 hora de repouso, foram realizadas as leituras em triplicata das absorbâncias em espectrofotômetro (Coleman 33 D) a $720 \mathrm{~nm}$. Utilizou-se como padrão o ácido gálico, nas concentrações de $2,5,10,15$ e $20 \mu \mathrm{g} / \mathrm{mL}$ para construir uma curva de calibração. A partir da reta obtida, realizou-se o cálculo do teor de fenólicos totais, expresso em mg de ácido gálico/ $100 \mathrm{~g}$ de amostra.

\section{Análise estatística}

Neste trabalho os resultados foram expressos como média \pm desvio-padrão. Para comparação das médias aritméticas, empregaram-se a análise de variância (ANOVA) e o teste Tukey, usando o software Prisma 4,0 (GraphPad). Adotou-se o nível de significância de 5\% de probabilidade $(\mathrm{p}<0,05)$.

\section{RESULTADOS E DISCUSSÃO}

Os resultados da composição centesimal e os valores nutricionais dos resíduos de polpas de frutas analisadas encontram-se na Tabela 1 .

A avaliação dos resíduos mostrou que, de forma geral, os resíduos possuem quantidades significativas dos macronutrientes (carboidratos, proteínas e lipídios), portanto, podem ser perfeitamente aproveitados para a alimentação, por meio de sua adição a outros produtos alimentícios.
Verificou-se que o teor de umidade de todos os resíduos de polpas de frutas avaliados neste estudo é elevado alcançando $93,86 \% \pm 0,08$ para o resíduo de cupuaçu (Theobroma grandiflorum). Vale ressaltar que o teor de umidade do resíduo de goiaba (Psidium Guayaba L.) $(65,54 \% \pm 0,32)$ foi estatisticamente diferente $(\mathrm{p}<0,05)$ e menor do que os demais.

O teor de cinzas encontrados nos resíduos de polpa de frutas analisados variou de $0,20 \% \pm 0,12$ para o resíduo de cupuaçu (Theobroma grandiflorum) a $0,72 \% \pm$ 0,02 para o resíduo de goiaba (Psidium Guayaba L.), portanto valores muito próximos, não havendo diferença estatística $(\mathrm{p}>0,05)$. Essas baixas taxas podem estar associadas a uma menor concentração dos minerais presentes nos resíduos analisados, visto que se tratavam de resíduos brutos, em que predominam altas concentração de água. Os resultados encontrados neste estudo estão próximos aos descritos na Tabela... (2009), para os frutos de acerola $(0,3 \%)$, graviola $(0,4 \%)$ e abacaxi $(0,53 \%)$.

O teor de proteínas dos resíduos de polpas de frutas analisados, variou de $0,56 \% \pm 0,03$ para o resíduo de bacuri (Platonia insigni) a 2,82\% $\pm 0,19$ para o resíduo de goiaba (Psidium Guayaba L.). As frutas de uma forma geral não são fontes potenciais de proteínas, entretanto parece que esse macronutriente se encontra predominantemente nas cascas e sementes. Uchôa et al. (2008) ao avaliar o resíduo de goiaba (Psidium Guayaba L.), encontraram $1,16 \%$ de proteína, valor inferior ao quantificado neste trabalho $(2,82 \%)$. Já Lousada Junior et al. (2005), analisando subprodutos de abacaxi (Ananas comosus L.), acerola (Malpighia glabra L.) e goiaba (Psidium Guayaba L.), encontraram valores elevados de proteínas, $8,4 \%, 10,5 \%$ e $8,5 \%$ respectivamente. Essas discrepâncias decorrem possivelmente pelas distintas partes que as indústrias de polpas de frutas utilizam para descarte como resíduo, além de fatores relacionados ao clima, solo, estágio de maturação dos frutos, dentre outros.

Tabela 1 - Composição nutricional dos resíduos de polpas de frutas tropicais.

\begin{tabular}{ccccccc}
\hline Resíduo & Umidade\% & Cinzas\% & Proteína\% & Lipídios\% & Carboidratos totais\% & Calorias (100 g) \\
\hline Goiaba & $65,54 \pm 0,32^{\mathrm{a}}$ & $0,72 \pm 0,02^{\mathrm{a}}$ & $2,82 \pm 0,19^{\mathrm{a}}$ & $2,94 \pm 0,01^{\mathrm{c}}$ & 27,98 & 150 \\
Acerola & $83,45 \pm 0,06^{\mathrm{b}}$ & $0,55 \pm 0,03^{\mathrm{a}}$ & $1,65 \pm 0,26^{\mathrm{b}}$ & $3,59 \pm 0,15^{\mathrm{d}}$ & 10,76 & 82 \\
Abacaxi & $88,19 \pm 0,80^{\mathrm{b}}$ & $0,53 \pm 0,04^{\mathrm{a}}$ & $1,05 \pm 0,01^{\mathrm{b}}$ & $0,69 \pm 0,03^{\mathrm{a}}$ & 9,54 & 49 \\
Graviola & $83,16 \pm 0,83^{\mathrm{b}}$ & $0,48 \pm 0,04^{\mathrm{a}}$ & $1,09 \pm 0,07^{\mathrm{b}, \mathrm{c}}$ & $2,28 \pm 0,13^{\mathrm{b}}$ & 12,99 & 77 \\
Bacuri & $85,81 \pm 0,13^{\mathrm{b}}$ & $0,65 \pm 0,28^{\mathrm{a}}$ & $0,56 \pm 0,03^{\mathrm{b}, \mathrm{c}}$ & $3,84 \pm 0,02^{\mathrm{e}}$ & 9,14 & 74 \\
Cupuaçu & $93,86 \pm 0,08^{\mathrm{b}}$ & $0,20 \pm 0,12^{\mathrm{a}}$ & $1,65 \pm 0,38^{\mathrm{c}}$ & $3,69 \pm 0,02^{\mathrm{d}}$ & 0,6 & 42 \\
\hline
\end{tabular}

a,b,c,d,e médias, na mesma coluna, seguidas de letras diferentes, diferem entre si pelo teste de Tukey a 5\% de probabilidade. 
Com relação ao conteúdo de lipídios, constatou-se que todos os resíduos apresentaram quantidades significativas desse nutriente, com exceção do resíduo de abacaxi, que apresentou apenas $0,69 \% \pm 0,03$. Já o resíduo de bacuri apresentou o maior teor lipídico $(3,84 \% \pm 0,02)$, sendo significativamente diferente dos demais $(\mathrm{p}<0,05)$. Os resíduos de acerola, e cupuaçu também apresentaram teores lipídicos expressivos com 3,59\% $\pm 0,15$ e 3,69\% $\pm 0,02$, respectivamente. Os resultados encontrados neste estudo estão próximos com os quantificados por Jerônimo et al. (2002), ao analisarem os bagaços de polpas de frutas de abacaxi (Ananas comosus L.) e acerola (Malpighia glabra L.) encontrando teores lipídicos de $0,54 \% \pm 0,01$ e 2,40\%, $\pm 0,03$, respectivamente. Já, Matias et al. (2005), ao avaliarem os resíduos de goiaba (Psidium Guayaba L.), obtiveram valores de $0,38 \% \pm 0,73$, portanto bem inferiores ao encontrados neste estudo que foi de $2,94 \% \pm 0,01$.

Quanto às concentrações de carboidratos totais observa-se uma variação muito grande nos resíduos avaliados, enquanto o cupuaçu (Theobroma grandiflorum) apresentou apenas $0,6 \%$, o resíduo de goiaba (Psidium Guayaba L.) apresentou $27,98 \%$, os resíduos de acerola (Malpighia glabra L.), abacaxi (Ananas comosus L.), graviola (Annona muricata L.) e bacuri (Platonia insignis) apresentaram valores maiores de $9 \%$. Como nos carboidratos totais estão incluídas as fibras alimentares, pode-se inferir que esses resíduos são boas fontes de fibras.

Outra variável analisada foi a quantidade de calorias. Com exceção do resíduo de goiaba (Psidium Guayaba L.) que apresentou $150 \mathrm{Kcal}$ por $100 \mathrm{~g}$, todos os outros resíduos apresentaram menos de $100 \mathrm{Kcal}$ por $100 \mathrm{~g}$ de resíduo, demonstrando que esse material possui baixo teor calórico, podendo ser utilizado como ingrediente na indústria de alimentos com o intuito de agregar valor e reduzir calorias.

Na Tabela 2, são mostrados os resultados das médias e desvio padrão dos teores totais de carotenóides, antocianinas, flavonóides, vitamina $\mathrm{C}$ e fenólicos totais nos diferentes resíduos de polpas avaliadas.

Os resíduos analisados apresentaram teores variáveis de carotenóides, destacando o resíduo de acerola (Malpighia glabra L.), com 881,56 $\pm 9,01 \mu \mathrm{g} / 100 \mathrm{~g}$ e o resíduo de goiaba (Psidium Guayaba L.) com 644,9 \pm 10,02 $\mu \mathrm{g} / 100 \mathrm{~g}$. Esses valores estão próximos das quantidades de carotenóides encontradas em alimentos que são considerados fontes potencias destes nutrientes, como milho $(880 \mu \mathrm{g} / 100 \mathrm{~g})$, mamão $(859 \mu \mathrm{g} / 100 \mathrm{~g})$ e pêssego $(651 \mu \mathrm{g} / 100 \mathrm{~g})$ (Godoy \& RodriguezAmaya, 1994).

Nos resíduos das polpas de frutas utilizados neste estudo os maiores valores de antocianinas totais foram encontrados na acerola $(8,84 \pm 0,02 \mu \mathrm{g} / 100 \mathrm{~g})$ e de goiaba $(3,2 \pm 0,34 \mu \mathrm{g} / 100 \mathrm{~g})$. Nos demais resíduos os valores foram muito baixos ou não detectados, como no caso do resíduo de abacaxi. Kuskoski et al. (2006), ao avaliarem as antocianinas totais em polpas de acerola e goiaba detectaram valores de 16,0 $\pm 0,5 \mu \mathrm{g} / 100 \mathrm{~g}$ e 2,7 $\pm 0,27 \mu \mathrm{g} / 100 \mathrm{~g}$. Esses mesmos autores não detectaram antocianinas nas polpas de abacaxi, graviola e cupuaçu.

Neste estudo, as concentrações de flavonóides variaram de $0,90 \pm 0,14 \mu \mathrm{g} / \mathrm{g}$ para o resíduo de abacaxi (Ananas comosus $L$ ) a 1,08 $\pm 0,05 \mu \mathrm{g} / \mathrm{g}$ para o resíduo de cupuaçu (Theobroma grandiflorum), valores inferiores aos de alimentos como laranja (9 $\mu \mathrm{g} / \mathrm{g})$, caju $(13 \mu \mathrm{g} / \mathrm{g}) \mathrm{e}$

Tabela 2 - Teores totais de carotenóides, antocianinas, flavonóides, vitamina C e fenólicos totais nos resíduos de polpas de frutas tropicais.

\begin{tabular}{|c|c|c|c|c|c|}
\hline Compostos & $\begin{array}{l}\text { Carotenóides } \\
(\mu \mathrm{g} / 100 \mathrm{~g})\end{array}$ & $\begin{array}{l}\text { Antocianinas } \\
(\mu \mathrm{g} / 100 \mathrm{~g})\end{array}$ & $\begin{array}{c}\text { Flavonóides } \\
(\mu \mathrm{g} / \mathrm{g})\end{array}$ & $\begin{array}{l}\text { Vitamina C } \\
(\mathrm{mg} / 100 \mathrm{~g})\end{array}$ & $\begin{array}{l}\text { Fenólicos Totais } \\
\text { (mg ácido gálico/ } \\
100 \mathrm{~g} \text { resíduo de } \\
\text { polpa de fruta) }\end{array}$ \\
\hline Goiaba & $644,9 \pm 10,02^{\mathrm{e}}$ & $3,2 \pm 0,34^{b}$ & $1,06 \pm 0,01^{\mathrm{a}}$ & $75,90 \pm 0,001^{\mathrm{e}}$ & $24,63 \pm 0,29^{\mathrm{d}}$ \\
\hline Acerola & $881,56 \pm 9,01^{\mathrm{f}}$ & $8,84 \pm 0,02^{\mathrm{c}}$ & $1,04 \pm 0,30^{\mathrm{a}}$ & $89,55 \pm 0,00^{\mathrm{f}}$ & $247,62 \pm 2,08^{\mathrm{e}}$ \\
\hline Abacaxi & $150,76 \pm 13,05^{\mathrm{c}}$ & ND* & $0,90 \pm 0,14^{\mathrm{a}}$ & $40,83 \pm 0,001^{\mathrm{c}}$ & $8,60 \pm 1,45^{\mathrm{b}}$ \\
\hline Graviola & $21,17 \pm 5,02^{\mathrm{a}}$ & $8,66 \cdot 10^{-2} \pm 3,05^{\mathrm{a}}$ & $1,03 \pm 0,22^{\mathrm{a}}$ & $64,35 \pm 0,001^{\mathrm{d}}$ & $18,60 \pm 0,80^{c}$ \\
\hline Bacuri & $222,80 \pm 6,75^{\mathrm{d}}$ & $3,31 \cdot 10^{-3} \pm 0,80^{b}$ & $0,95 \pm 0,20^{\mathrm{a}}$ & $35,71 \pm 0,00^{\mathrm{b}}$ & $8,57 \pm 0,09^{b}$ \\
\hline Cupuaçu & $127,9 \pm 4,54^{\mathrm{b}}$ & $3,06 \cdot 10^{-3} \pm 0,50^{b}$ & $1,08 \pm 0,05^{\mathrm{a}}$ & $14,47 \pm 0,001^{\mathrm{a}}$ & $4,66 \pm 0,40^{\mathrm{a}}$ \\
\hline
\end{tabular}

*ND - Não Detectado.

a,b,c,d,e,f médias, na mesma coluna, seguidas de letras diferentes, diferem entre si pelo teste de Tukey a 5\% de probabilidade. 
polpa de acerola $(23 \mu \mathrm{g} / \mathrm{g})$. Esses alimentos possuem reduzido teor de flavonóides se comparados com a alface crespa $(195 \mu \mathrm{g} / \mathrm{g})$, cebola roxa $(390-660 \mu \mathrm{g} / \mathrm{g})$ e couve (266-399 $\mu \mathrm{g} / \mathrm{g}$ ), entretanto, os dados descritos por diferentes autores para flavonóides são muito variáveis (Huber \& Rodriguez-Amaya, 2008). Vale ressaltar que, apesar dos teores de flavonóides em alimentos serem determinados geneticamente, fatores como estação do ano, clima, composição do solo, estágio de maturação, preparo, processamento e estocagem dos alimentos influenciam diretamente em tais concentrações (Huber \& Rodriguez-Amaya, 2008).

Quanto aos flavonóides e antocianinas tem-se que os dados acerca dos mesmos, principalmente em alimentos são ainda insuficientes mesmo a nível mundial. Essa carência é ainda mais acentuada no Brasil. Assim, a quantificação de tais compostos é extremamente relevante para estudos futuros.

Quanto à vitamina $\mathrm{C}$, ao comparar os valores encontrados nesses resíduos com a ingestão diária recomendada para adultos (Institute of Medicine - IOM, 2000), que estabelece um teor de $75 \mathrm{mg}$ para mulheres e $90 \mathrm{mg}$ para homens, observa-se que os resíduos, com exceção do cupuaçu, bacuri e abacaxi, podem ser considerados fontes importantes de vitamina $\mathrm{C}$ atingindo a ingestão diária recomendada.

A determinação dos fenólicos totais mostrou que a maioria dos resíduos de polpas de frutas apresentaram quantidades variáveis destes compostos, com destaque para a polpa de acerola com 247,62 $\pm 2,08 \mathrm{mg} / 100 \mathrm{~g}$ de fenólicos totais, exibindo o mais elevado teor destes constituintes, sendo estatisticamente diferentes dos demais ( $\mathrm{p}<0,05)$. Em seguida, vem o resíduo de polpa de goiaba com 24,63 \pm 0,29 mg/100 g. O resíduo de polpa de graviola apresentou valores intermediários de compostos fenólicos totais e os resíduos de bacuri e cupuaçu apresentaram os menores valores desse constituinte.

Comparando os teores de fenólicos totais dos resíduos de goiaba, abacaxi e graviola com as polpas de frutas de goiaba (Psidium guayava), com 83,1 mg/100 g; abacaxi (Ananas sativa), com 21,7 mg/100 g e graviola (Anona muricato), com 84,3 mg/100 g), pode-se observar que os teores dos resíduos são inferiores (Kuskoski et al., 2005).

\section{CONCLUSÕES}

Os resíduos industriais das polpas de frutas utilizados neste estudo apresentaram quantidades variáveis de macronutrientes, apresentando, de uma forma geral, elevado teor de água e reduzido teor de calorias, destacando-se as polpas de goiaba, acerola e cupuaçu como as maiores fontes de proteínas e as polpas de cupuaçu, bacuri e goiaba como as maiores fontes de lipídeos. Quanto aos constituintes antioxidantes se destacam as polpas de acerola e goiaba como fontes potenciais de carotenóides totais, fenólicos totais e vitamina C. Este estudo abre a perspectiva de novos trabalhos com a finalidade de desenvolvimento de novos produtos alimentícios onde possam ser adicionados esses resíduos, agregando valor nutricional e antioxidante e diminuindo a contaminação ambiental por resíduos industriais.

\section{REFERÊNCIAS BIBLIOGRÁFICAS}

ABRAHÃO, S.A.; PEREIRA, R.G.F.A.; DUARTE, S.M.da S.; LIMA, A.R.; ALVARENGA, D.J.; FERREIRA, E.B.

Compostos bioativos e atividade antioxidante do café

(Coffe arabica L.). Ciência e Agrotecnologia, Lavras, v.34, n.2, p.414-420, mar./abr., 2010.

\section{ASSOCIATION OF OFFICIAL ANALYTICAL CHEMISTS. Official Methods of Analysis of AOAC International. 17.ed. Arlington, 2000. v.2.}

COSTA, R.P.; MENENDEZ, G.; BRICARELLO, L.P.; ELIAS, M.C.; ITO, M. Óleo de peixe, fitosteróis, soja e antioxidantes: impactos nos lipídios e aterosclerose. Revista da Sociedade de Cardiologia, São Paulo, v.10, n.1, p.819-832, 2000.

FERREIRA, F.A.G.; GRAÇA, M.E.S. Tabela de composição de alimentos portugueses. 2.ed. Lisboa: Instituto Superior de Higiene Dr. Ricardo Jorge, 1983.

GODOY, H.T.; RODRIGUEZ-AMAYA, D.B. Occurrence of cis-isomers of provitamin A in Brazilian fruits. Journal of Agricultural and Food Chemistry, Washington, v.42, n.6, p.1306-1313, 1994.

HUBER, L.S.; RODRIGUEZ-AMAYA, D.B. Flavonóis e flavonas: fontes brasileiras e fatores que influenciam a composição em alimentos. Alimentos e Nutrição, Campinas, v.19, n.1, p.97-108, 2008.

INSTITUTE OF MEDICINE. Dietary reference intakes. Whashington, National Academy, 2000.

INSTITUTO ADOLF LUTZ. Métodos físico-químicos para análise de alimentos. 4.ed. São Paulo, 2005. 
JERÔNIMO, C.E.M.; MAGALHÂES, C.G.; SANTIAGO JÚNIOR, A.F.; OLIVEIRA, V.G.; MELO, H.N.S.

Caracterização dos resíduos das indústrias potiguares de beneficiamento de polpas de frutas. In:

ASSOCIAÇÃO BRASILEIRA DE ENGENHARIA

SANITÁRIA EAMBIENTAL. Desafios ambientais da globalização. Vitória, 2002. p.1-7.

KIM, Y.; GIRAUD, D.W.; DRISKELL, J.A. Tocopherol and carotenoid contents of selected Korean fruits and vegetables. Journal of Food Composition and Analysis, Netherlands, v.20, n.6, p.458-465, 2007.

KUSKOSKI, E.M.; ASUERO, G.A.; MORALES, M.T.; FETT, R. Frutos tropicais silvestres e polpas de frutas congeladas: atividade antioxidante, polifenois e antocianinas. Ciência Rural, Santa Maria, v.36, n.4, p.1283-1287, 2006.

KUSKOSKI, E.M.; ASUERO, G.A.; TRONCOSO, A.M.; MANCINI-FILHO, J.; FETT, R. Aplicación de diversos métodos químicos para determinar actividad antioxidante em pulpa de frutos. Revista de Ciência e Tecnologia de Alimentos, Campinas, v.25, n.4, p.726-732, 2005.

LOUSADA JUNIOR, J.E.; NEIVA, J.N.N.; RODRIGUEZ, N.M.; PIMENTEL, J.C.M.P.; LÔBO, R.N.B. Consumo e digestibilidade de subprodutos do processamento de frutas em ovinos. Revista Brasileira de Zootecnia, Viçosa, v.34, n.2, p.659-669, 2005.

MARTINS, C.R.; FARIAS, R.M. Produção de alimentos x desperdício: tipos, causas e como reduzir perdas na produção agrícola. Revista da Faculdade de Zootecnia, Veterinária e Agronomia, v.9, n.1, p.83-93, 2002.

MATIAS, M.F.O.; OLIVEIRA, E.L.; GERTRUDES, E.; MAGALHÂES, M.A. Use of fibres obtained from the cashew (Anacardium ocidentale, $L$ ) and guava (Psidium guayava) fruits for enrichment of food products. Brazilian Archives of Biology and Technology, Curitiba, v.48, p.143-150, 2005.
MELO, E.A.; MACIEL, M.I.S.; LIMA, V.A.G.L.; NASCIMENTO, R.J. Capacidade antioxidante de frutas. Revista Brasileira de Ciências Farmacêuticas, São Paulo, v.44, n.2, p.193-201, 2008.

PIENIZ, S.; COLPO, E.; OLIVEIRA, V.R.de; ESTEFANEL, V.; ANDREAZA, R. Avaliação in vitro do potencial antioxidante de frutas e hortaliças. Ciência e Agrotecnologia, Lavras, v.33, n.2, p.552-559, mar./abr., 2009.

SCHIEBER, A.; STINTZING, F.C.; CARLE, R. Byproducts of plant food processing as a source of functional compounds: recent developments. Trends Food Science Technology, Cambridge, v.12, n.11, p.401413, 2001.

SENA, R.F.; NUNES, M.L. Utilização de resíduos agroindustriais no processamento de rações para carcinicultura. Revista Brasileira de Saúde e Produção Animal, Bahia, v.7, n.2, p.94-102, 2006.

SWAIN, T.; HILLS, W.E. The phenolic constituents of Punnus domestica: iquantitative analysis of phenolics constituents. Journal of the Science of Food and Agriculture, London, v.19, n.1, p.63-68, 1959.

TABELA brasileira de composição de alimentos. Disponível em: <http://www.unicamp.br/nepa/taco/sy. Acesso em: 20 fev. 2010.

UCHÔA, A.M.A.; COSTA, J.M.C.; MAIA, G.A.; SILVA, E.M.C.; CARVALHO, A.F.F.U.; MEIRA, T.R. Parâmetros físico-químicos, teor de fibra bruta e alimentar de pós alimentícios obtidos de resíduos de frutas tropicais. Segurança Alimentar e Nutricional, Campinas, v.15, n.2, p. $58-65,2008$.

VASCONCELOS, S.M.L.; SILVA, A.M.; GOULART, M.O.F. Pró-antioxidantes e antioxidantes de baixo peso molecular oriundos da dieta: estrutura e função. Nutrire, São Paulo, v.31, n.3, p.95-118, 2006. 